\title{
A Study of Logic Model Based on Web
}

\section{Application}

\author{
Xiaoguang $\mathrm{An}^{1, \text { a }}$, Pengfei Liu ${ }^{1, \mathrm{~b}}$ \\ ${ }^{1}$ Department of Information Engineering Jilin Business and Technology \\ College, Changchun, China \\ 334233682@qq.com, ${ }^{b} 1257120205 @ q q . c o m$
}

\begin{abstract}
The study starts with the characters of Web application, then puts forward the concept, basic idea and method of construction of Web application logic model, after that the logic model obtained through the designed algorithm and the performance of algorithm achieves the ideal effect. It can be used to detect users in time for various operation of Web application through Web-based application logic model, and judge whether the requests from users for resources is legal, if not, invasion will be taken into account.
\end{abstract}

Key words: Web Application Logic Model; Security Mechanism; Intrusion Detection

\section{Introduction}

With the vigorous development of Internet, the number of connecting to the Internet Host and Internet users also increase explosively. The rapid development of Internet will also bring a negative impact that is the Internet security problems exposed constantly. Although many scholars have done lots of research work in the field of information security, the present Internet still has security problems to be solved. Web application logic model is put forward in this paper, which can 
process the data obtained from capturing information section, then mainly done on the system of Noise Link, process of link repetition information and carry on a correct judgment of link address to store the information, finally to capture the new data information. The security of Internet can be effectively guaranteed.

\section{Logic Model of Web Application}

The way of communication between Web client and server, and the process of user access are shown in Figure 1: the client accesses data requests, first to the Web server (e.g.IIS, Internet Information Server), then through a Web server to the database server, and the return of the data is just opposite of the above. The flow of the information is among browser, Web server and database server.

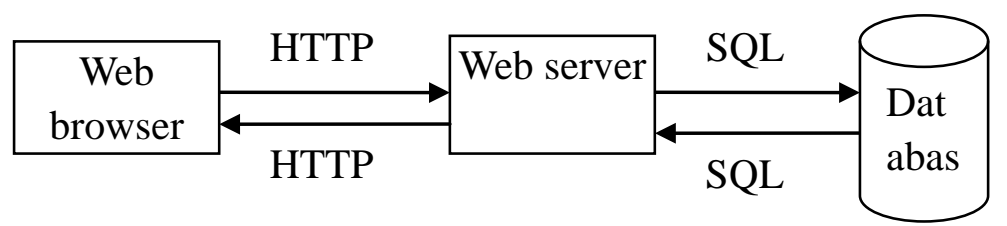

Figure 1: Client-server

\section{Introduction of Web Application Logic Model}

It is used to achieve the building of Web application logic model, through putting forward hyper-linked structure algorithm based on breadth traversal.

\section{Grammatical Analysis}

HTML can be analyzed and extracted valuable link information, so it can not be regarded as common Character Stream. It has its own morphology and semantics. Therefore the analysis should be according with its morphology and semantics, which to the greatest degree to meet the requirements of accuracy and 
comprehensiveness.

Here is the expression of HTML grammar rules (BNF). This expression gives the lexical definition of HTML. After the lexical analysis of Web pages through HTMLBNF and analysis of all the HTML elements and properties, it can be deeply analyzed its syntax and semantics, then extracted the useful information.

HTML BNF*:

HTML $=*($ Element[Paragraph])

Element=Starting $\mid$ Ending

Starting="<" * (Blank)Name* (Blank)[ * (Attribute* (Blank))]”>”

Ending="<" * (Blank)"/”Name* (Blank)">”

Attribute=Name[* (Blank)”=” * (Blank)AttrValue]

AttrValue=DBQuotValue|SgQuotValue|NoQuotValue

Paragraph $=*$ (ParaChar)

DBQuotValue="<>* (DbQuotChat $)<>$ "

SgQuotValue=",'” * (SgQuotChar),',"

NoQuotValue $=*$ (NoQuotChar)

Name $=1 *$ (NameChar)

Definition of terminal symbol:

Blank=”” | ”|t” | “'n” | “|r”

ParaChar $\in$ Charset-“<”

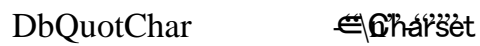

SgQuotChar

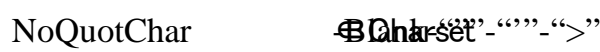


NameChar=HiAlpha | loAlpha

HiAlpha=”A" |”B”|... | "Z”

LoAlpha="a”|"b”|... |"z"

Charset::=all characters in the character set used in document collection(Charset )

Thereinto, *string constants is enclosed by" "; quotation mark indicates <’>; $n *$ indicates the latter contents is repeated $n$ times or more (n the default is 0 ); [ ] used to enclose options.

The grammar described above is the standard of regular grammar, because it can be analyzed by constructing Finite Automation. The work of this grammar analysis can be arranged for a separate module to complete, meanwhile it is responsible for analysis of Web page and extracting all the elements and their properties. Through HTML grammar analysis, the elements and contexts in it can be extracted orderly and then be analyzed, it is called analysis of HTML tokens flow. And the analysis is to get next mark of tokens flow by constant analysis. This analysis procedure can be completed by designing separate modules, we can design various separate modules to complete different tasks of analysis. As Figure 2 shows, a specific module specializes in analyzing the link information appeared in $<\mathrm{a}>$, and the other module specializes in analyzing link information appeared in $<$ frame $>$. 


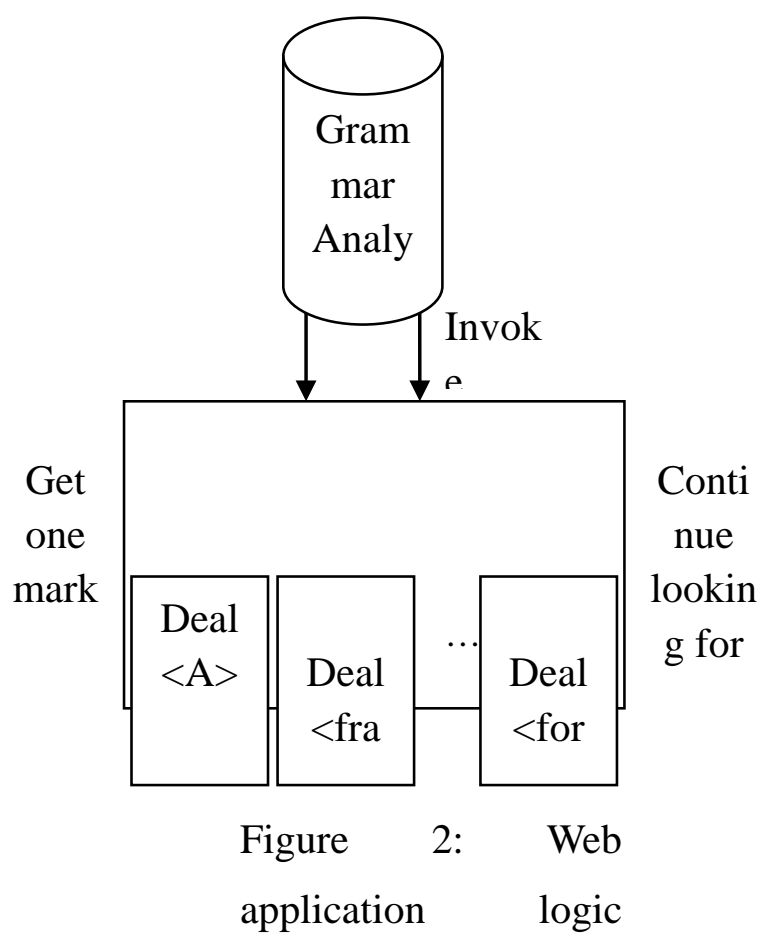

Analysis of grammar is introduced in the design process of logic model, it is also the key technology used in constructing this model, and ensure the accuracy of the design of this logic model.

\section{Description of Algorithm}

In order to make more clear description of algorithm, the concept of link layer is introduced to help with the specific implementation and related description of algorithm, so that it can make further understanding between each link on the macro level.

Algorithm:link layer is the distance,with distance to 1 scale, between link and home page of Web application. If one of the links in the Web application named AO,with layer 3, and if it includes another link A1 in the page that based on link 
address of $\mathrm{AO}$, then the layer of $\mathrm{A} 1$ is 4 . Concrete examples are shown in Figure3, a link relations.

In Figure 3, if A is the home page of Web application, so by the available that A layer of 0 , and B,C,D layer of 1,E,F,G,H layer of 2 .

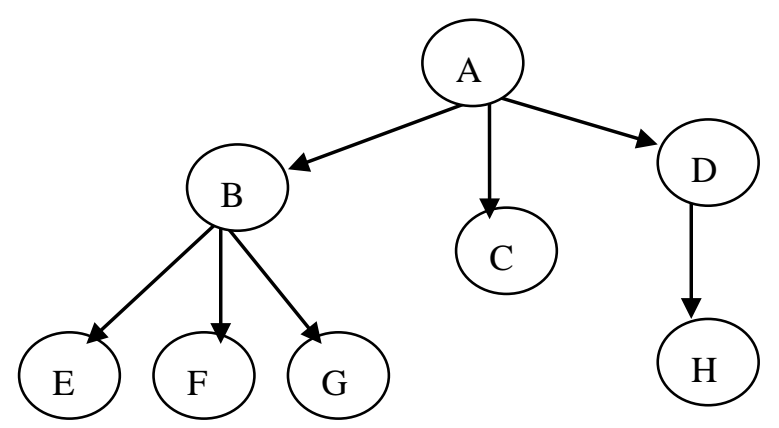

Figure 3: Link relation

The algorithm of link layer reflects the relations among links to a certain extent, and incorporates the size of a certain distance linked to the mouth of Web application.

Main ideas of algorithm:starting from the entrance of a given Web site, using Get method of HTTP request to obtain the HTML document, and defining related regular grammar to analyze HTML, then storing the corresponding information of link relations in database, traversing all the link information of this layer, then continuing traversing link to next layer until all the link information of Web site can be traversed.

\section{Applications of Web-based Application Logic Model}

The Web application logic model can be used to transform link relations among link addresses as a state, and make the page elements in Web page form, which is corresponding to link address, as the parameter among the conversion of state, 
that is making page of Web application as state in logic model, and make link relations of pages as state to transform, make related page elements of pages as parameter of state transformation; the parameter depends on concrete conditions of mutual transformation among states.

We take state as peak, take the link relations among pages as directed edge, then we can get a directed graph of Web application logic model, in the meanwhile, we finally get logical model of the Web application based on hyper-linked structure, the Web application logic model can also implement the software running in the process of legitimate users access to the safety of the system resources and prevent the illegal invasion of the visitors.

\section{Conclusion and Prospect}

In this paper, the author starts from the properties of Web application, puts forward the concept of Web application logic model, gives out the expressions and related algorithm rules, and studies its incidence matrix. Then the model is constructed and made effective comparison between the operation access behaviors and datum gained from server and information from Web application logic model, the security model system can detect the various operations of Web application done by users in time, and judge whether the request of resources is legal. If not, intrusion will be taken into account.

It is far from satisfying for adopting the hyper-linked structure algorithm based on breadth traversal on the level of accuracy, through building logical model of Web application to study the security of Web application, so it is still needed to 
integrate comprehensive factors in order to get more accurate logical model. It will be the context of further research in the future.

\section{Acknowledgements}

The authors gratefully acknowledge the fund of this study by Jilin province talent development project in 2013 (20131079) and the Jilin province science and technology department soft science research project (20130420026FG) "Study of Jilin province engineering and technology talent support for strategy emerging industry”.

\section{References}

[1] Sun Xijin and Huang Yinghui. Semantic Web Application: Properties and Development Model [J]. Computer Application \& Software, Nov. 2012.

[2] Yang Yanjie. Study on Key Techniques about Web Application Test Model [J]. Manufacturing Automation, Sept. 2012.

[3] Li Xinlong. Semantic Web Application Development Method and Example Analysis [J]. Computer Technology \& Development, Sept. 2013.

[4] Wang Xin. Research on Key Technologies of Web Application System Security Detection [J]. Beijing University of Posts and Telecommunications, May 2011.

[5] Yue Qing and Hao Baoshui. Study of SQL Injection Defense Based on Web Application [J]. Public Science \& Technology, Apr. 2011. 